\title{
Intrapartum Hypoxic Damage is detected by Hypoxia Index to Prevent Cerebral Palsy
}

\author{
Kazuo Maeda* \\ Honorary Professor, Department of Obstetrics and Gynecology, Tottori University Medical School, Yonago, Japan \\ *Corresponding Author: Kazuo Maeda, Honorary Professor, Department of Obstetrics and Gynecology, \\ Tottori University Medical School, Yonago, Japan, Tel: 81-859-22-6856; E-mail: maedak@mocha.ocn.ne.jp
}

Received: 26 February 2018; Accepted: 26 March 2018; Published: 28 March 2018

\begin{abstract}
Aims: To prevent fetal brain damage and cerebral palsy (CP).

Methods: The hypoxia index was studied to solve the vague late deceleration (LD). Hypoxic fetal brain damage was known by the loss of variability. Early delivery before the loss of variability will prevent cerebral palsy, however, no numeric sign of the loss of variability was present, which was seeked by the author in the summing hypoxic effect in repeated FHR deceleration, where the sum of durations of FHR deceleration (min) was divided by the lowest FHR (bpm) and multiplied by 100, in the hypoxia index (HI), in the full FHR monitoring course, possibly using a computer.
\end{abstract}

Results: The Hi was 25 \& 26 in CP and severe brain damage, while 20-24 in abnormal FHR but neither brain damage nor cerebral palsy with significant differences. Thus, a numeric threshold in HI to develop cerebral palsy was 25, and the safe HI is 24 or less. The CP will be prevented by the early delivery when the HI is less than 25.

Discussion: As HI was 6 in 3 connected LD while HI was 26 after 50 min's LD repetition, the outcome depends on the repetition, but not to the LD pattern, and all of early, late, variable decelerations and continuous fetal bradycardia are evaluated by HI, namely, HI decides outcome, instead of deceleration pattern, where outcome is digitally diagnosed using numeric HI.

Conclusion: HI covers the role of all decelerations, while FHR pattern was discarded in computerized FHR diagnosis.

Keywords: FHR; CTG; Actocardiogram; Acceleration; Variability; Hypoxia Index; Brain Damage; Cerebral Palsy 


\section{Introduction}

As the fetus is covered by amniotic fluid, uterine muscle, and maternal abdominal wall, its medical study was almost impossible in the past, where mother's perception of fetal movement and enlarging abdomen were the subjects to estimate fetal well- being. Stethoscopic auscultation was introduced to listen to fetal heart sound after 20 weeks pregnancy.

The author started his medical engineering from maternal electroencphaogram [1] and neonatal respirogram [2].

Fetal QRS was detected by maternal limb leads using Seitengalvanometer in old time. Maternal abdominal surface electrode detected amplified fetal QRS in 1940s. Also maternal abdominal microphone detected fetal heart sound and listened in the labor. Hon EH invented intrapartum fetal scalp lead fetal electrocardiogram (FECG) then he applied it to measure peak to peak interval converting it into fetal heart rate (FHR) traced on running chart, with amniotic pressure recording uterine contraction, then reported fetal heart rate pattern describing early, late, mild and severe variable decelerations in 1960 .

Hon's EFM prepared the combination of fast FECG recorder with FHR+intrauterine pressure on the slow chart recorder. Caldeyro-Barcia, recorded FECG \& fetal heart rate with a needle electrode through abdominal wall, with uterine contraction by intramyo- metrial pressure, and found Type 1 and 2 dips in fetal heart rate, where Type 2 dip was the same as Hon's late deceleration. Type 2 developing mechanism was the compression of iliac arteries with acontracted pregnant uterus, resulting the los of maternal blood flow through placenta, which was confirmed by pelvic arterial angiography, while Hon reported the LD simply as placental insufficiency.

Hammacher K, Germany, used Kardio-toko-gram composed of a fetal phonographic microphone to record FHR curve and external tokodynamometer without membrane rupture in pregnancy. Pattern classification was similar to two pioneers, but he emphasized FHRbaseline variability, of which loss was "silent", which was severely abnormal FHR.

Maeda's Asia first fetal monitor was an cardio- tocogram which use a microphone or scalp electrode, amniotic pressure or guard-ring type exernal tocodynamoeter, namely external FHR monitoring was possible for the diagnosis in pregnancy.

\section{Methods}

\subsection{Cardiotocogram}

We studied fetal inrapartum states with FHR and uterine contraction curves recorded on the slow chart (cardiotocogram, CTG), visually detecting FHR changes. The first CTG, provided 1964, recorded FHR by fetal heart tone fetal microphone triggering fetal heart rate meter in external CTG, though it was more noisy than the fetal scalp lead FECG, while the needle electrode of scalp lead was risky to viral infection from the mother [5]. 
The external fetal monitoring changed to ultrasound Doppler autocorrelation FHR meter 1974 (Takeuchi Y \& Hogaki), of which CTG was as clear as the FHR recorded by scalp lead FECG. Thus, the fetal heart tone was changed to ultrasonic autocorrelation fetal heart rate meter, which is main use in external CTG at present.

However, the FHR deceleration classification with visual recognition into periodic and variable changes followed by early, late and variable decelerations [6] were subjective resulting vague decision and inter-observer difference, and also the pattern classification was difficult to explain developmental mechanism of FHR changes, e.g. no physiologic sinusoidal pattern was separated from pathologic one, the origin of heart rate variability was vague, variable decelerations were explained by nervous reflex in mild deceleration and hypoxia in severe variable one, i.e. they were explained by two different principles.

\subsection{Quantitative FHR score evaluation}

The author discarded visual pattern classification, and quantitatively analyzed FHR deceleration, baseline FHR and numeric data of decelerations was objectively evaluated using FHR score in 1969 [4] (Figure 1, Table 1), rejecting vague analysis and inter-observer difference.

Firstly, the FHR change was analyzed into composd elements, including the baseline FHR (mean of the most FHR data in every 10 bpm steps), amplitude of long term variability, number of decelerations in 5 min, duration of every deceleration, every amplitude, every nadir heart rate, every lag time from the contraction peak, every recovery time to the baseline, presence of acceleration accompanied the deceleration (Figure 1), percentage of Apgar score lower than 7 in the parameter, and each evaluation score 1-4 determined by the percentage of lower Apgar score than 7 in the statistics of births. All evaluation scores were summarized in the 5 minutes to achieve FHR score in the 5 minutes.

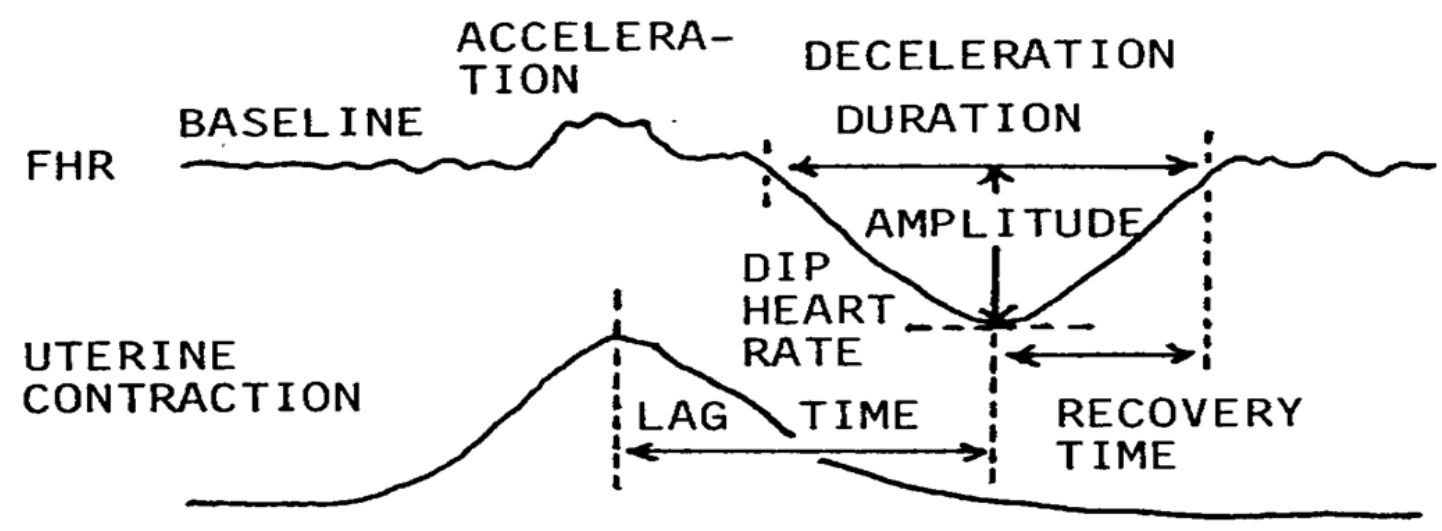

Figure 1: Each deceleration and FHR baseline were analyzed by quantitative data. It looks troublesome, but it is easy in computerized determination. 
The FHR score and expected abnormal Apgar score and UApH calculated by regression equations were obtained and reported attending doctor directly and rapidly in case of computerized FHR monitoring.

\begin{tabular}{|l|c|c|}
\hline FHR signs & Incidence of Apgar $<7$ (\%) & Evaluation score \\
\hline FHR baseline $<110$ or $>180 \mathrm{bpm}$ & 70 & 3 \\
\hline Deceleration duration $>1$ min & 71 & 3 \\
\hline Nadir heart rate $<100 \mathrm{bpm}$ & 37 & 2 \\
\hline Nadir amplitude $>50 \mathrm{bpm}$ & 50 & 2 \\
\hline Lag time $>40 \mathrm{~s}$ & 71 & 3 \\
\hline Recovery time $>40 \mathrm{~s}$ & 63 & 2 \\
\hline No acceleration accompanied & 45 & 4 \\
\hline W- shape and the loss of variability & 100 & 2 \\
\hline
\end{tabular}

Table 1: Evaluation scores were set by the low Apgar score incidence in each FHR signs. The sum of evaluation scores in $5 \mathrm{~min}$ is FHR score. The fetus was abnormal if FHR score was 10 or more, and severely abnormal if the score was 20 or more.

Fetal outcome was expected by the application of regression equations calculated between FHR score and neonatal Apgar score and UApH. ( Apgar score was calculated by the equation of hypoxia index and Apgr score too). The results are reported doctors directly and quickly by cell phone or LAN with technical as well as clinical terms, e.g. FHR score and Apgar score (Table 2).Thus, electronic data was changed to medical terms to a quick understanding of doctor to facilitate clinical correspondence in the decision of treatment in the busy obstetric works. The electronic to medical conversion was tried.

\begin{tabular}{|c|c|c|}
\hline FHR score & $\mathbf{1 0}$ & $\mathbf{1 5}$ \\
\hline Apgar score & 6 & 4 \\
\hline UApH & 7.2 & 7.08 \\
\hline Asphyxia & mild & moderate \\
\hline
\end{tabular}

Table 2: Calculations with regression equations Apgar score and UApH are expected by FHR score even in early stage of labor, alarming doctor in computer monitoring, namely: FHR score $=\mathrm{X}$, Apgar score $=\mathrm{Y}$ and $\mathrm{UApH}=\mathrm{Z}$; $\mathrm{Y}=$ 9.361-0.33 X; Z=7.31-0.016X

Also, Apgar score is expected by the hypoxia index as follows; Hypoxia index= X; Apgar score=Y; Y=10.37-0.28X

\begin{tabular}{|c|c|c|}
\hline Hypoxia Index & $\mathbf{1 0}$ & $\mathbf{2 6}$ \\
\hline Apgar score & 8 & 3 \\
\hline Asphyxia & No & severe \\
\hline
\end{tabular}




\subsection{Actocardiogram (ACG)}

Fetal movement was recorded with spikes, fetal heart rate (FHR) and uterine contraction on the ACG [9] (Figure 2), in which FHR changed by fetal movements via fetal brain function, thus, various problems of CTG were solved, e.g. FHR acceleration was provoked by fetal movements (Figure 2), thus, the loss of acceleration was early sign of hypoxia, physiologic sinusoidal heart rate was separated from pathologic one by its synchronization to periodically changing movements [10], and FHR variability (LTV) was provoked by minor fetal movements due to fetal brain function, therefore, the loss of variability was the sign of fetal brain damage [11,12]. As an actocardiograph records uterine contraction at the same time, the machine is acto-cardio-toco-graph, namely, the device can be used as an actocardiogram and also cardiotocogram according to the intention of its user.

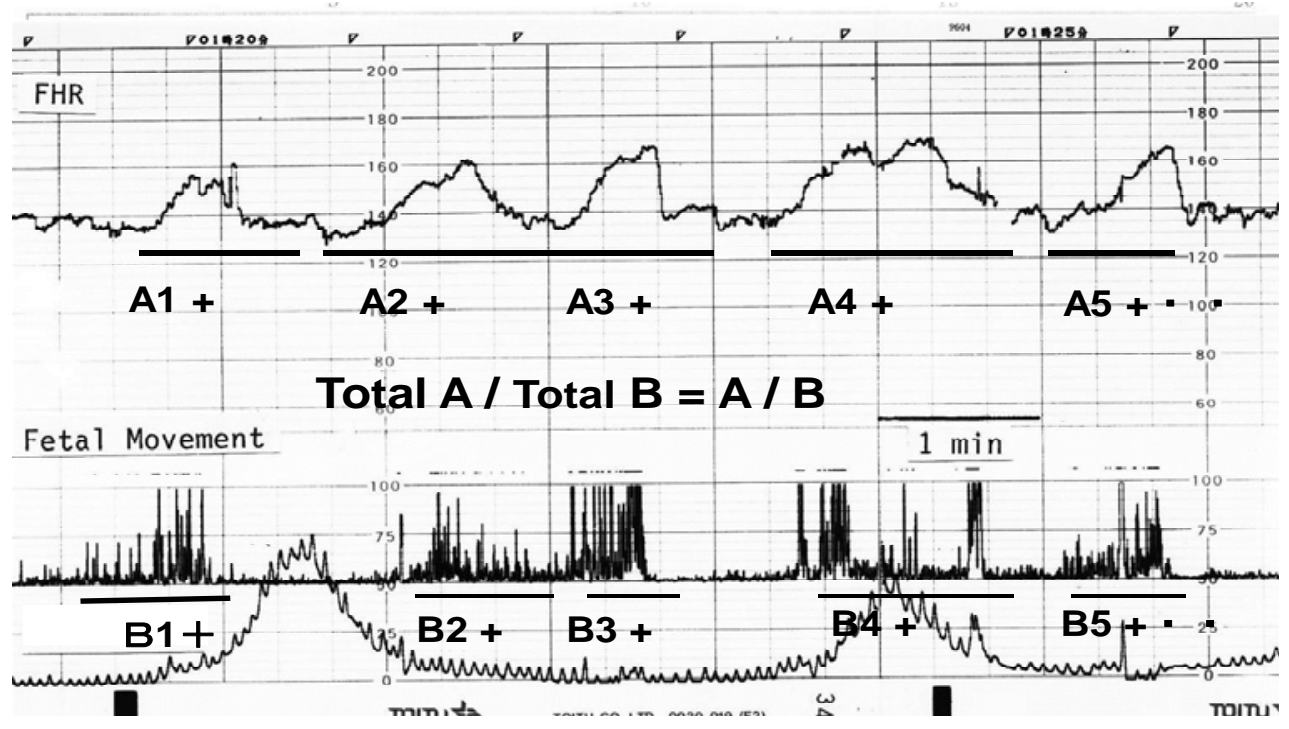

Figure 2: Triangular FHR accelerations were associated with the clusters of fetal movement spikes (movement bursts) in active fetal state. FHR acceleration duration (the sum of A) ratio to the movement duration (sum of B) is the A/B ratio, which predicts fetal outcome [16].

FHR changes were provoked by the reaction of fetal mid- brain to fetal movement with 7 sec delay due to the presence of integral circuit function of the brain, which were proved by electronic and physiologic simulations. The acceleration was lost in early hypoxic state preserving FHR variability. The variability was lost by the most severe hypoxia forming totally flat baseline such as that of fetal anencephaly, i.e. the loss of variability indicated the severe fetal brain damage, followed by cerebral palsy.

Therefore, the fetus is recommended to deliver before the loss of variability [12] which were preceded by the loss of acceleration and decreased variability. Severe bradycardia, and high FHR score are also the sign to precedes brain 
damage, and high Hypoxia Index higher than 24 indicate severe fetal brain damage followed by cerebral palsy. Thus, neonatal brain damage followed by cerebral palsy will be prevented by the early C-delivery before the loss of variability [11,12] which will be preceded by high hypoxic index of 25 or more. The decrease of neonatal CP before introduction oh hypoxia index will be explained by early caesarean delivery decided by a severe FHR change in fetal monitoring. However, there will be no $\mathrm{CP}$ if the $\mathrm{HI}$ is lower than 24 at delivery at present.

Physiologic sinusoidal FHR is separated from pathologic ominous sinusoidal, if the sinusoidal FHR change is synchronized with the periodic changes of fetal movements, which was mainly fetal respiratory movements [10].

Fetal hiccupping movements are continuously repeated sharp spikes with 2 sec intervals for more than 10 min, which did not accompany FHR acceleration, while there was no hypoxia, because it is an independent convulsive diaphragmatic actions without fetal brain control. It is proved by continuous sharp spikes with 2 sec intervals without formation of movement burst, but only continuous fetal movements with 2 sec intervals in app.10 minutes (Figure 3). Further to accurate diagnosis of fetal hiccupping will be done by real-time ultrasound B-mode imaging, where the fetal body suddenly extends within half second and repeats the sharp motion every 2 sec continuously for 10 minutes. That is very characteristic show if compared the other fetal movements.
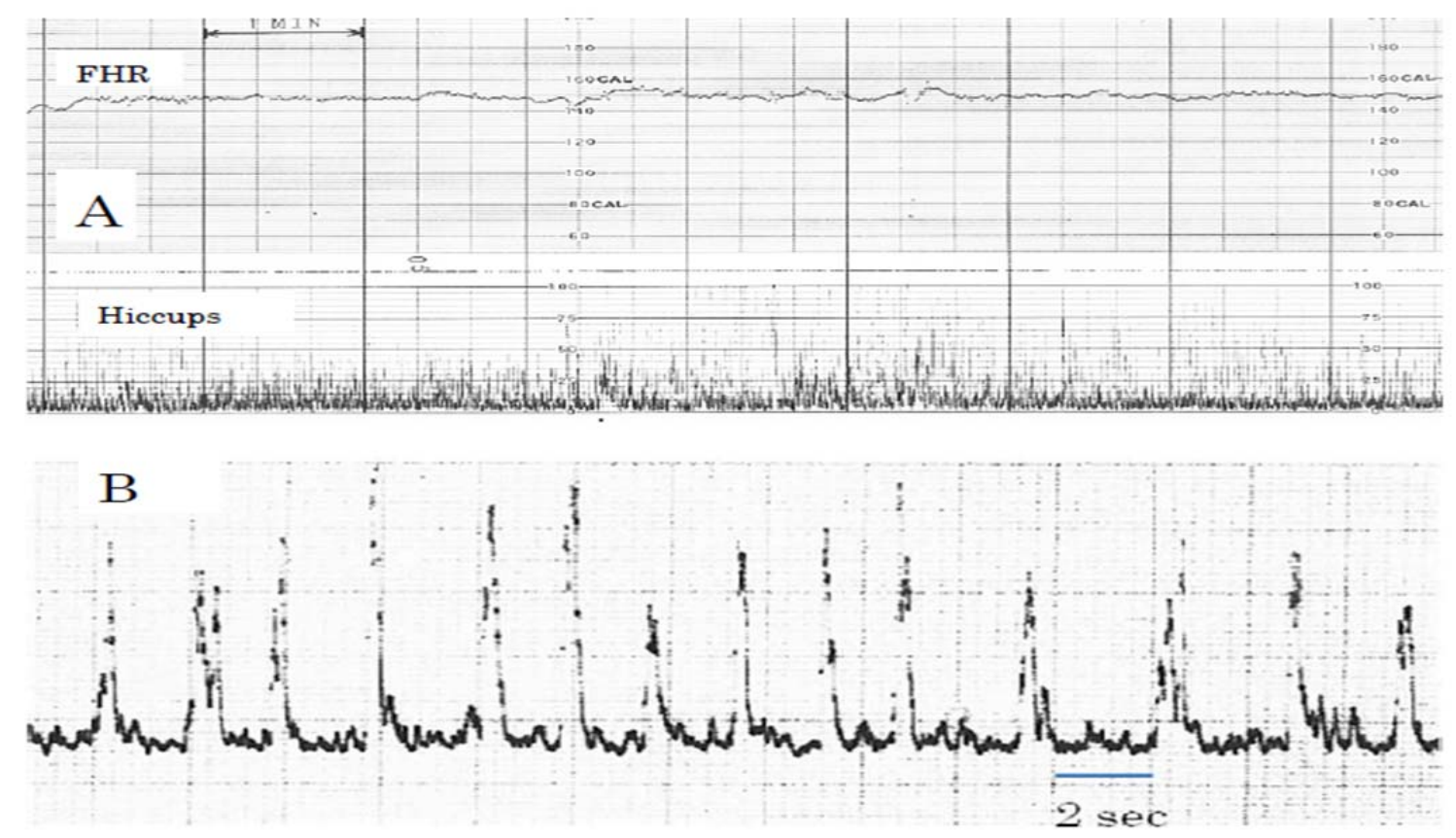

Figure 3: Actocardiograms of fetal hiccupping movements. A: Usual $3 \mathrm{~cm} / \mathrm{min}$ chart speed, Continuous sharp spikes without movement burst. B: Fetal hiccuping record with rapid chart speed, where spike is very sharp and its interval was regularly 2 sec, There is no movement burst. 


\subsection{Hypoxia Index}

It was a controversy that fetal outcome was favorable after 3 connected late decelerations (LDs), though it is reported that LD is ominous [6], while highly repeated LDs lost variability, Apgar score was 3, and the infant was severe brain damage in the author's experience. In addition, LD is defined after the repetition for 15 min in some reports. It may mean that the LD must be defined after 6 decelerations, if the deceleration appears twice in 5 minutes, namely, LD is evaluated after repetition of 6 decelerations, namely, repetition is important in LD.

Thus, the author estimated that the hypoxia damages the fetus when the sum of deceleration is large, namely, hypoxic effect of 3 decelerations is too weak to be hazardous. Thus, the sum of duration (min) of decelerations was divided by the lowest nadir FHR (bpm), which is the intensity of hypoxia, then multiplied by 100, where the hypoxia index is obtained, where a deceleration will be the same as the dip area. The heart rate is used instead of $\mathrm{PaO}_{2}$, because rabbit $\mathrm{PaO}_{2}$ closely correlates heart rate when the $\mathrm{PaO}_{2}$ is lower than 50mmHg [3], and human fetal $\mathrm{PaO}_{2}$ is less than 50 mmHg [4]. Thus, completed Hypoxia Index is as follows;

Hypoxia Index (HI) = Sum of deceleration duration (min) divided by the lowest FHR (bpm), and multiplied by 100 .

The HI was measured in cases of the loss of variability followed by fetal brain damage and cerebral palsy, to determine the threshold to develop cerebral palsy (CP), where HI was 25 in a case of the loss of variability followed by CP, and 26 in repeated LD for 50 min, whose Apgar was 3, and the 3 month infant died in brain hemorrhage. Thus, upper limit of HI not to be cerebral palsy is lower than 25. Actually, the HI was 20 to 24, smaller than 25, in cases of abnormal FHR, who associated neither the loss of variability nor cerebral palsy (Table 3). Therefore, HI should be lower than 25 at delivery to prevent cerebral palsy [11, 12]. Also, the problem of controversy LD will be solved, because the authors case of three connected LDs’ HI was 6 and the A/B ratio was 1.3 (larger than 1.0) which was normal range, and its Apgar score was 9, while the outcome was ominous when the deceleration was repeated 50 min to increase HI to 26, thus, deceleration durations are summarized in HI.

\begin{tabular}{|l|c|c|}
\hline & $\begin{array}{c}\text { The loss of variability, fetal } \\
\text { brain damage and cerebral } \\
\text { palsy }\end{array}$ \\
\hline & Yes & No \\
\hline $\mathrm{HI}=25$ or more & 2 & 0 \\
\hline $\mathrm{HI}=0-24$ & 0 & 5 \\
\hline
\end{tabular}

$\mathrm{P}=0.048<0.05$, significant difference

Table 3: There was a significant difference in the Chi square test of two hypoxia index groups. 


\subsection{Maternal posture}

As late deceleration [8], the deceleration in supine hypotension and umbilical cord compression disappeared when the mother changed her posture to lateral from supine, because LD is caused by the compression of iliac artery by contracted uterus, followed by the loss of placental maternal circulation $[7,8]$, the mother is recommended to change her posture to lateral one from supine with the purpose to remove FHR deceleration.

\subsection{Fetal brain damages in preterm birth infants}

Yamamoto et al reported the periventricular echo density (PVE) in preterm fetuses, which was highly echogenic periventricular zone in B-mode diagnosed by high GLHW, and $18 \%$ of the PVE turned into neonatal brain PVL followed by CP, when the PVE lasted during pregnancy until preterm delivery [13].

Two strategies are proposed to prevent the brain damage; 1) Since neither PVL nor CP was found in neonates after full term birth [13], the pregnancy is prolonged until term birth by any effective tocolysis, one of which will be pharmaceutical, and another will be the sedation of uterus-brain nerve in the positive feed-back loop of labor contraction by anesthetic procedure [15].

2) As the PVE lasted until preterm delivery, PVE will be found in the neonatal brain immediately after preterm birth, which is detected by ultrasound in preterm neonate, then detected PVE will be treated before the change into PVL, namely, such neonatal brain repairing material as the growth factor will be administered to the case of neonatal PVE, because the growth factor disappeared within 3-4 days after birth in normal neonates. The proposals will be heavily studied in the future before practical application..

\section{Results}

\subsection{FHR score}

FHR score was calculated manually in 1969, while mainly computerized calculation was common in the present.

Apgar score and UApH was predicted using regression equations as follows;

Apgar score is 7 or more, if FHR score is $<10$

Apgar is 6, if FHR score $=10$

Apgar is 4 and UApH 7.08, acidosis, if FHR score = 15,

Apgar is 2 \& acidosis, if FHR score $=20$.

Attending doctor is alarmed by the objective expetqtion..

The doctor will decide early delivery according to expected Apgar score and pH. 


\subsection{Actocardiogram}

Developing a mechanism of normal and pathological FHR changes were clarified by the analysis of actocardiogram, particularly, FHR acceleration, variability and their pathologic changes in hypoxia, namely, acceleration and variability are the reaction of the fetal brain to fetal movements, thus, their disappearance means the suppression or damage of fetal brain due to hypoxia, and the fetus is cured by early delivery before the loss of variability. A physiologic sinusoidal FHR is differentiated from pathologic one, the progress of fetal hypoxic damage is predicted by hypoxia index, and fetal behavior is known by actocardiogram.

\subsection{Hypoxia Index}

Controversy late deceleration was clarified by the application of HI. Intrapartum fetal brain damage followed by infantile cerebral palsy will be prevented when the fetus receives early delivery before the HI reaches 24 which is the highest limit not to develop the loss of variability followed by fetal brain damage and cerebral palsy.

\section{Discussion}

Fetal heart rate diagnosis with FHR pattern classification into early, late, or variable decelerations was changed to objective numeric FHR score and hypoxia index, which covers the roles of various FHR deceleration patterns, including early, late, or variable decelerations. The addition of fetal movement in actocardiogram clarified fetal brain response to fetal movement, the process to hypoxic FHR changes, or developing mechanism of physiologic sinusoidal FHR, etc.

\section{Conclusion}

Intrapartum hypoxic fetal brain damage followed by cerebral palsy will be detected and prevented by the application of novel hypoxia index, instead of subjective FHR pattern classification. Numeric analysis of FHR changes, including FHR score, baseline variability, sinusoidal FHR, hypoxia index and so on will be incorporated in FHR analyzing computer.

\section{References}

1. Maeda K. Electroencephalographic studies on eclampsia and preeclampsia, J Obstet Gynecol Res 42 (2016):11-20.

2. Maeda K. Invention of neonatal respirograph. Obstetrics Gynecology World 6 (1954)527-528.

3. Umezawa J. Studies on the relation between heart rate and $\mathrm{PaO}_{2}$ in hypoxic rabbit: a comparative study for fetal heart rate change during labor. Acta Obstet Gynecl Jpn 28 (1976): 1203-1212.

4. Maeda K, Kimura S, Nakano H, et al. Pathophysiology of Fetus. Fukuoka Printing, Fukuoka, 1969.

5. Maeda K, Cosmi E, Gardosi J et al. FIGO News, Intrapartum surveillance: recommendations on current practice and overview of new developments. Intern J Gynecol Obstet 49 (1995): 213-221.

6. Hon EH. An Atlas of Fetal Heart Rate Patterns. Harty Press, 1968, New Haven.

7. Caldeyro-Barcia R. Poseiro JJ, Mendez-Bauer C, Gulin LO. Effect of abnormal uterine contractions on fetal 
Obstet Gynecol Res 2018; 1 (1): 009-018

DOI: 10.26502/ogr002

heart rate in labor 1967: 9-27.

8. Poseiro, JJ.Mendez-Bauer C, Caldeyro-Barcia R, etal. Effect of uterine contractuions on maternal blood flow through the placenta. Perinatal factors affectinghuman development. Paho Advisary Committee 1969: $161-171$.

9. Maeda K. Invention of ultrasonic Doppler fetal actocardiograph and continuous recording of fetal movements. J Obstet Gynecol Res 42 (2016): 5-10.

10. Ito T, Maeda K, Takahashi H, et al. Differentiation between physiologic and pathologic sinusoidal FHR pattern by fetal actocardiogram. J Perinat Med 22 (1994): 39-43.

11. Maeda K. Origin of the long-term variability and acceleration of FHR studied for the prevention of cerebral palsy in fetal hypoxia and general insults. J Perinat Med 42 (2014): 401-403.

12. Maeda K. Modalities of fetal evaluation to detect fetal compromise prior to the development of significant neurological damage. J Obstet Gynecol Res 40 (2014): 2089-2094.

13. Yamamoto N, Utsu M, Maeda K, et al. Neonatal periventricular leukomalacia preceded by fetal periventricular echodensity. Fetal Diag Ther 15 (2000): 198-208.

14. Maeda K. A proposal to reduce congenital cerebral palsy. J Health Med Inform 4 (2013): 135.

15. Maeda K. Developing process of regular labor contraction. Edelweiss Apli Sci Tech 2 (2018): 118-119.

16. Maeda K, Iwabe $\mathrm{T}$, Ito $\mathrm{T}$, et al. Detailed multigrade evaluation of fetal disorders with the quantified actocardiogram. J Perinat Med 37 (2009): 392-396.

Citation: Kazuo Maeda. Intrapartum Hypoxic Damage is detected by Hypoxia Index to Prevent Cerebral Palsy. Obstetrics and Gynecology Research 1 (2018): 009-018.

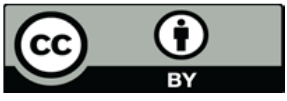

This article is an open access article distributed under the terms and conditions of the Creative Commons Attribution (CC-BY) license 4.0 